\title{
Leg Bone Fracture Segmentation and Detection using Advanced Morphological Techniques
}

\author{
K.Sudha Rani, K.Mani Kumari, Ganti Amulya, Eswar pothineni, velagapudi Pavani, P.Susvitha \\ Reddy
}

\begin{abstract}
The bone fracture is the most common problem and is likely to occur due to traumatic incidents like vehicle accidents, sporting injuries or due to conditions like osteoporosis, cancer related to bones. Fracture cannot be viewed by naked eye and so $X$-ray, CT, ultrasound, MRI images are used to detect it. These images cannot be diagnosed directly and henceforth image processing plays a very important role in fracture detection. This paper presents an image processing technique that uses Laplacian method of edge detection for accurate identification of fractured bone area from the $\mathrm{X}$-ray/CT images. From the fractured bone area several parameters like mean, standard deviation are calculated in order to analyze the accuracy and sensitivity of the used technique. NIVISION assistant software is used and the statistical parameters are calculated.
\end{abstract}

Keywords: Segmentation, Histogram, Morphological operations.

\section{INTRODUCTION}

The human skeleton is the interior substructure of the body consisting of 206 bones. A broken bone is a fracture and is very common problem in India. The common sites for bone fracture are the wrist, leg, hand, hip etc. Fractures are usually expounded by their location, alignment of bones, if there are any associated complications with blood and nerve function and if the skin is intact at the injury site. For bone fracture detection there are many processing techniques [11]. Image segmentation is one of the most important stages in fracture detection which is done in order to express the image as a physically meaningful connected region. Analysis various features such as edge, texture, colour, brightness and so on can be achieved [12]. Few image segmentation techniques are Fuzzy C-Means segmentation, Edge Tracking and gradient-based techniques, and the Circle Hough Transform technique. Reference [14] states that high sensitivity and good outputs can be obtained by using Edge tracking

Revised Manuscript Received on July 10, 2019.

K.Sudha Rani, 1 Associate Professor, VNR VJIET, Pragathi Nagar, Hyderabad,Telangana-90, India.

(email : sudharani_k@vnrvjiet.in)

K.Mani Kumari, Vardhaman College of Engineering, Nagarguda

Shamshabad Road, Kacharam, Hyderabad, Telangana 501218.India.

(email : joemani23@gmail.com)

Ganti Amulya, UG Student, VNR VJIET, Pragathi Nagar,

Hyderabad,Telangana- 90, India.

(email :ganti.amulya50@gmail.com )

Eswar pothineni, UG Student, VNR VJIET, Pragathi Nagar,

Hyderabad,Telangana- 90, India.

(email : eswarpothineni13@gmail.com)

velagapudi Pavani, UG Student, VNR VJIET, Pragathi Nagar,

Hyderabad,Telangana- 90, India.

(email : pavanivelagapudi53@gmail.com)

P.Susvitha Reddy, UG Student, VNR VJIET, Pragathi Nagar, Hyderabad,Telangana- 90, India. technique.

The author Tandeep Kaur 1.et.al. in the paper entitled as "Bone Fracture Detection using Image Segmentation"proposed a method that can detect bone fracture automatically. This method uses segmentation, Fuzzy c-means and multilevel wavelet algorithms. An efficient algorithm based on thresholding and morphological operators is explained .The results are obtained with $89.6 \%$ accuracy and $93.1 \%$ precision with the sensitivity of the fracture detection being equal to $95.5 \%$.

The author San Myint2.et.al.in the paper"Detecting Leg Bone Fracture in X-RAY Images" presented an image processing technique to detect bone fracture. This proposed system uses canny edge detection method for segmentation and Hough transform technique for line detection in image. The three main steps involved in the system are pre-processing, segmentation and fracture detection.

The author Luis Nascimento3.et.al.in the paper titles as "Computer-Aided Bone Fracture identification based on ultrasound images" presented a new method for computer-aided identification of bone fracturesbased on finding bone lines in ultrasound images. The suggested strategy involves noise reduction followed by bone line identification and lastly identification of possible fracture on the bone line. The results state that $89 \%$ of the fractures (within 44 images) were accurately diagnosed.

The author Kamil Dimililer4.et.al.in the paper "Intelligent Bone Fracture Detection System" developed an intelligent classification system that is capable of detecting bone fractures. This system uses back propagation neural networks and comprises of two stages where in the first stage involves processing of images and the next stage is the classification phase. The inferences state that, by using SIFT algorithm $91.4 \%$ of the images were correctly yielded and by using BPNN $94.3 \%$ accuracy is obtained.

Theauthor Malashree5.et.al. in the paper "Automatic Detection of Radius of Bone Fracture"proposed an algorithm that uses Hough transform for automation detection of radius of bone fracture. The system involves three principle steps, image pre-processing, segmentation, feature extraction and detection of radius of bone fracture. MATLAB 2013 is used as the programming tool. This technique shows about $90 \%$ accuracy (within 20 images). 
The author Dhiraj B.Bhakare6.et.al. in the paper entitled as "A Novel Approach for Bone Fracture Detection using Image processing"presented a computer based analytic techniques for the detection of bone fracture using X-ray or CT images. This technique starts from pre-processing followed by edge detection, segmentation, image classifier and finally fractures detection. The proposed method is accurate up to $85 \%$.

The author Harmanpreet Kaur7.et.al. in the paper "Detection of Fractures in Orthopedic X-Ray Images" explained a procedure to perceive the fracture of bone from an input image. This proposed work uses edge detection, intensity based segmentation, and frequency transformation based methods. Fuzzy C-mean clustering algorithm is applied on image.

The author Cephas Paul Edward8.et.al. in the paper entitled as "A Robust Approach For Detection Of the type Of Fracture from X-Ray Images" suggested an innovative approach to detect fractures. Different edge detection methods like Sobel Operator, Laplacian of a Gaussian and canny, prewitt operators can be applied on the X-Ray image. In this paper a novel edge detection algorithm is also explained, inorder to analyse the results quality metrics were also used.

The authorA.V.Manjari9.et.al. in her paper "Detection and Classification of Bone Fractures Using GLCM Features" explained about the detection of bone fractures and the various stages involved in it. On the X-Ray images first pre-processing techniques are applied followed by edge detection using canny edge detection algorithm .Segmentation is performed by K-means clustering

Algorithm and finally feature extraction is achieved using GLCM feature extraction algorithm and are classified based on Region of Interest algorithm.

The author Ozgur Ozturk10.et.al. in the paper " Detection of bone fractures using image processing techniques and artificial networks" proposed an artificial neural network for fracture detection. For image segmentation K-means was used and the presented technique has $89 \%$ success rate.

The author Oishila Bandyopadhyay13.et.al. in the paper "Long-Bone Fracture Detection in Digital X-ray Images Based on Concavity Index" presented a technique for automatic fracture detection for long- bone X-ray images based on digital geometry. This is achieved by tracing bone contour in an X-ray image and using a novel concept of concavity index of the contour for identification of bone fracture locations.

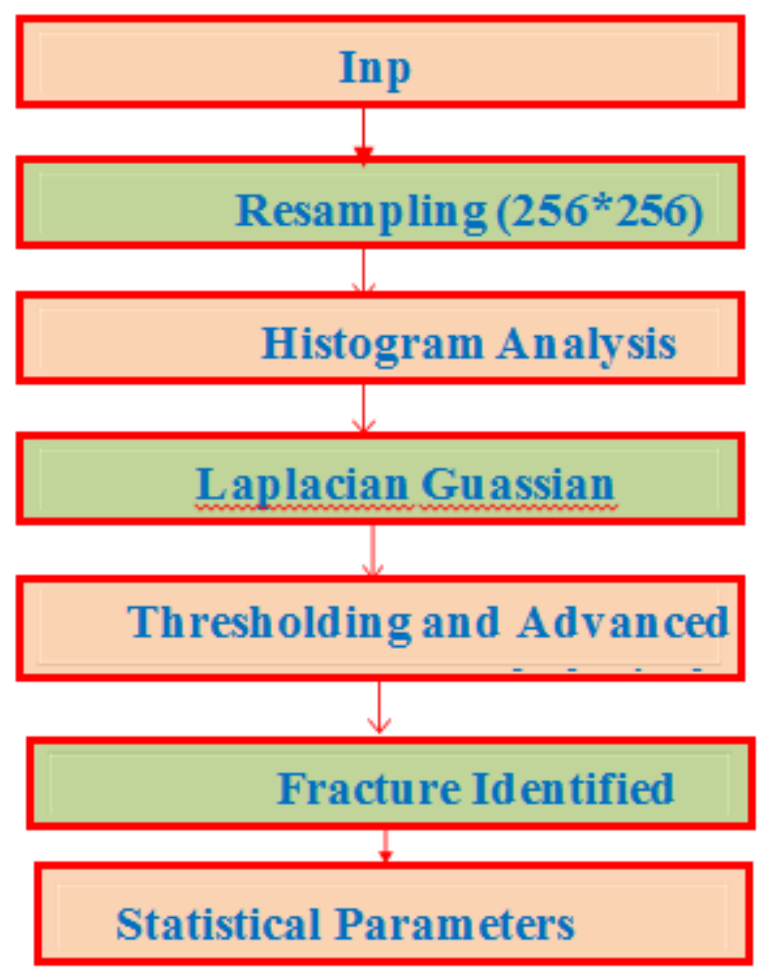

Fig.1 Flow chart for Leg fracture detection

\section{METHODOLOGY}

The given image is resampled to $256 \mathrm{X} 256$ by using the colour plane extraction in vision Assistant software. Resampling of the image is a technique used in order to convert the original image to an image with different width and/or height. Let $\mathrm{G}(\mathrm{x}, \mathrm{y})$ be the original image and $\mathrm{N}(\mathrm{x}, \mathrm{y})$ be the predefined resolution of the sampled image. Then the corresponding relation is given as:

$\mathrm{N}(\mathrm{x}, \mathrm{y})=$ Height $^{*}$ width $=256 * 256$ pixels. (1)

The images that undergo resampling yield images having fixed geometrical dimensions with the resolution being the same.

\section{Histogram:}

Histogram Analysis is then done on the resampled image. Histogram is the graphical representation of pixel intensity (taken on $\mathrm{x}$-Axis) versus number of pixels (taken on y-Axis). Let us consider 8-bit grayscale image. For this image 256 different intensities are possible and the histogram of this image will display 256 numbers graphically representing the distribution of pixels among these values only.

\section{Laplacian-Guassian filter:}

To find regions of rapid change in images derivative filters like laplacian filters are used. Before applying this filter it is 
important to smooth the image as laplacian filter is sensitive to noise. This is done by using Guassian filter. Hence forth the image undergoes a two-step process called as Laplacian of Guassian operation and the mathematical equation is given as:

$$
\mathrm{L}(\mathrm{a}, \mathrm{b})=\nabla^{2} f(a, b)=\frac{\delta^{2} f(a, b)}{\delta a^{2}}+\frac{\delta^{2} f(a, b)}{\delta b^{2}}
$$

In morphological image processing erode function is one of the basic operations that can be applied to both binary and as well as gray scale images. Gray -scale erosion of a point is defined as the minimum number of points in its neighbourhood that define the structuring element.If $a(x)$ denotes an image, $\mathrm{b}(\mathrm{x})$ is the grayscale structuring element, the grayscale erosion of a by $b$ is mathematically represented as:

$$
(f \Phi \mathrm{b})(\mathrm{x})=\inf _{y \in B}[\mathrm{f}(\mathrm{x}+\mathrm{y})-\mathrm{b}(\mathrm{y})]
$$

\section{RESULTS \& DISCUSSIONS}

After applying advanced morphological technique, the image is quantified and finally the required parameters are determined.

The fig (a) is the applied image to which colour plane extraction and histogram analysis is done. After the application of Laplacian-Guassian filter it yields an image $1 \mathrm{~b}$, from which the parameters like standard variation, mean, minimum and maximum values are calculated.

The treatment required to cure medical illness always relies on diagnosis tests. Henceforth the accuracy of these plays a crucial role. To explicate diagnosis test specificity, accuracy, and accuracy are widely used statistics. The following are the common terms used to explain the concepts of specificity, accuracy and sensitivity related to disease diagnosis.

Let us consider, positive $=$ determined and negative $=$ rejected

True Positive (TP): Number of cases that correctly determine people with bone fracture as fracture

True Negative (TN): Number of cases that correctly determinehealthypersons as healthy

False Positive (FP): Number of cases that incorrectly determine healthy persons as people with fracture.

False Negative (FN): Number of cases that incorrectly determine people with fracture as healthy

Accuracy of a test is the ability to recognize the patient and healthy cases correctly. It can also be defined as the proportion of true positive and true negative in all the cases evaluated.

Mathematically it is given as:

$$
\text { Accuracy }=\frac{T P+T N}{T P+T N+F P+F N}
$$

Sensitivity is the ability to identify sick patients who are suffering with the disease. It is the proportion of true positive subjects in a total group of subjects with disease. Mathematical expression is given as follows:

$$
\text { Sensitivity }=\frac{T P}{T P+F N}
$$

Specificity is the ability to identify healthy cases correctly. It represents the probability of negative test result in a subject without disease.

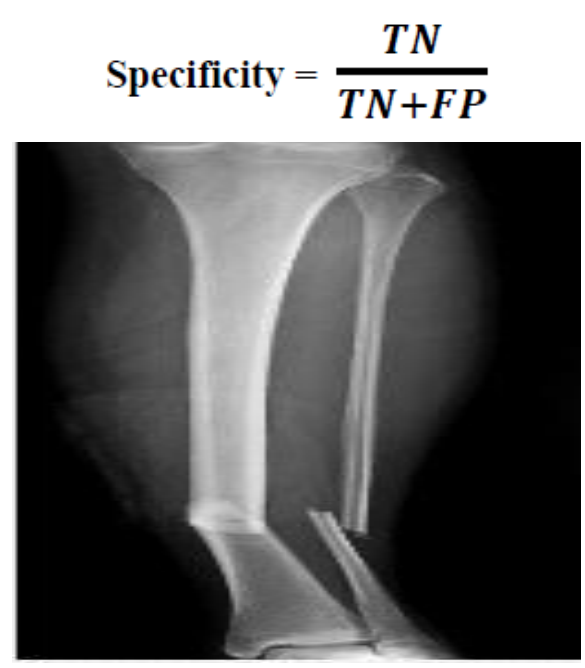

Fig: Input image (a)

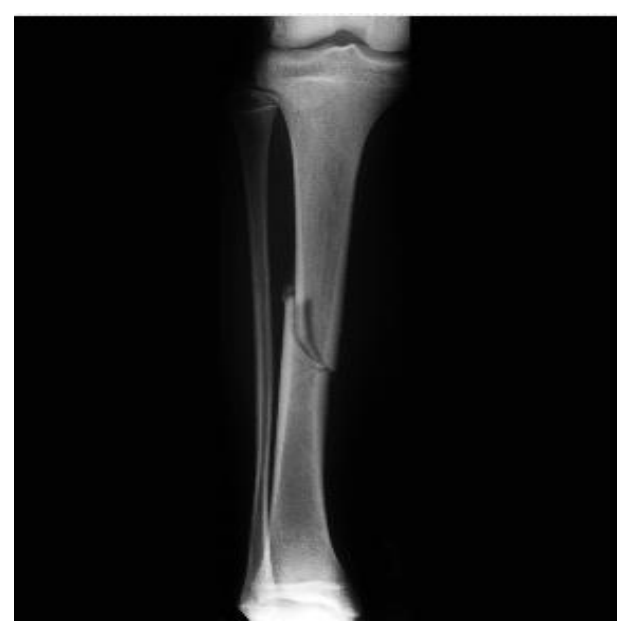

(b) Fractured leg 
Table 1 Different patient's information

\begin{tabular}{|c|c|c|c|c|c|c|c|c|c|c|c|c|c|c|}
\hline $\overrightarrow{0}$ & 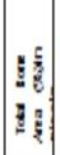 & 告 & 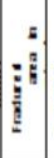 & $\mid$ & 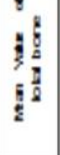 & إن & 迸 & & & 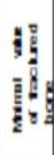 & 3 & i⿱ & 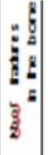 & $\hat{v}$ \\
\hline$p 1$ & 428 & 11.3 & \begin{tabular}{|l|}
0.7 \\
\end{tabular} & 0.2 & 0.7 & 6 & 60.8 & 40.7 & 0.0 & 180 & 254.0 & 189.0 & 1.0 & \\
\hline$p 2$ & 349 & 9.2 & 0.5 & 0.1 & 45.3 & 63.7 & 73.4 & 86.4 & 0.0 & 00 & 255.0 & 255.0 & 1.0 & 1.3 \\
\hline p3 & 489 & 12.4 & \begin{tabular}{|l|}
0.6 \\
\end{tabular} & 0.2 & 56.8 & 61.3 & 82.1 & 87.9 & 0.0 & 00 & 255.0 & 255.0 & 1.0 & 1.2 \\
\hline \multirow[t]{2}{*}{$p 4$} & 256 & 6.8 & \begin{tabular}{|l|}
0.7 \\
\end{tabular} & 0.2 & 31.2 & 49.8 & 55.0 & 79.2 & 0.0 & 00 & 255.0 & 255.0 & 2.0 & 2.9 \\
\hline & & 6.8 & 1.1 & 0.3 & & 63.6 & & 80.1 & & 00 & & 255.0 & & 4.4 \\
\hline$p 5$ & 356 & 9.4 & 0.9 & 0.2 & 45.4 & 79.5 & 78.1 & 110.1 & 0.0 & 00 & 255.0 & 255.0 & 1.0 & 2.4 \\
\hline \multirow[t]{2}{*}{$p 6$} & 301 & 8.8 & 1.0 & 0.3 & 3 & 37.6 & 46.2 & 56.7 & 0.0 & 00 & 55.0 & 255.0 & 2.0 & 3.2 \\
\hline & & 8.8 & 0.6 & 0.2 & & 27.3 & & 41.8 & & 00 & & 251.0 & & 1.8 \\
\hline p7 & 456] & 12.1 & $\mid 1.9$ & 0.5 & 174.6 & $\begin{array}{c}185 . \\
2\end{array}$ & 42.2 & 48.8 & \begin{tabular}{|c|}
40. \\
0
\end{tabular} & 540 & 253.0 & 243.0 & 1.0 & 4.1 \\
\hline p8 & 507 & \begin{tabular}{|l|}
13.4 \\
\end{tabular} & 1.4 & 0.4 & 39.3 & 48.7 & 63.1 & 72.5 & 0.0 & 00 & 55.0 & 255.0 & 1.0 & 2.7 \\
\hline$p 9$ & 41.7] & \begin{tabular}{|l|l|}
11.0 \\
\end{tabular} & \begin{tabular}{|l|}
1.5 \\
\end{tabular} & 0.4 & 48.2 & 46.1 & 72.8 & 69.7 & 0.0 & 00 & 255.0 & 255.0 & 1.0 & 3.7 \\
\hline p10 & 221 & \begin{tabular}{|l|}
5.8 \\
\end{tabular} & 0.3 & 0.1 & 49.2 & 75.2 & 82.7 & 104.1 & 0.0 & 00 & 255.0 & 255.0 & 1.0 & 1.5 \\
\hline p11 & $37 / 3$ & 9.9 & 1.9 & 0.5 & 21.7 & 26.3 & 36.3 & 40.1 & 0.0 & 00 & 255.0 & 244.0 & 1.0 & 5.1 \\
\hline$p 12$ & 206 & 5.4 & 0.8 & 0.2 & 47.5 & 64.3 & 75.1 & \begin{tabular}{|l|}
88.4 \\
\end{tabular} & 0.0 & 00 & 255.0 & 255.0 & 1.0 & 4.0 \\
\hline p13 & 186 & 4.9 & 1.2 & 0.3 & 36.3 & 39.9 & 61.9 & 70.6 & 0.0 & 00 & 255.0 & 255.0 & 1.0 & 6.7 \\
\hline p14 & 149 & 4.0 & 0.4 & 0.1 & 28.4 & 21.9 & 52.2 & 38.3 & 0.0 & 00 & 255.0 & 255.0 & 1.0 & 2.8 \\
\hline$p 15$ & 162 & 4.3 & 0.4 & 0.1 & 10.3 & 11.2 & 10.8 & 8.6 & 0.0 & 00 & 92.0 & 49.0 & 1.0 & 2.8 \\
\hline p16 & 168 & \begin{tabular}{|l|}
4.4 \\
\end{tabular} & \begin{tabular}{|l|}
0.8 \\
\end{tabular} & 0.2 & 67.8 & 57.6 & 93.1 & 89.1 & 0.0 & 00 & 255.0 & 255.0 & 1.0 & 5.0 \\
\hline p17 & 84 & 2.2 & 0.2 & 0.0 & 36.0 & 48.4 & 36.2 & 43.1 & 0.0 & 00 & 200.0 & 150.0 & 1.0 & 2.2 \\
\hline \multirow[t]{2}{*}{$p 18$} & 313 & 8.3 & 1.0 & 0.3 & 24.3 & 25.6 & 48.5 & 46.4 & 0.0 & 00 & 255.0 & 255.0 & 2.0 & 3.2 \\
\hline & & 8.3 & 0.6 & 0.2 & & 23.5 & & 37.3 & & 00 & & 255.0 & & 2.0 \\
\hline \multirow[t]{2}{*}{ p19 } & 159 & 4.2 & 0.3 & 0.1 & 43.0 & 51.1 & 71.9 & 84.8 & 0.0 & 00 & 255.0 & 255.0 & 2.0 & 1.7 \\
\hline & & \begin{tabular}{|l|}
4.2 \\
\end{tabular} & 0.5 & 0.1 & & 55.5 & & 93.1 & & 00 & & 255.0 & & 3.3 \\
\hline 20 & 158 & 4.2 & 0.4 & 0.1 & 45.7 & 67.9 & 73.4 & 86.4 & 0.0 & 00 & 255.0 & 255.0 & 1.0 & 2.5 \\
\hline \multirow[t]{2}{*}{$p 21$} & 136 & 3.6 & \begin{tabular}{|l|}
0.2 \\
\end{tabular} & 0.1 & 3 & 39.5 & 25.9 & 17.6 & 0.0 & 70 & 57.0 & 79.0 & 2.0 & 1.4 \\
\hline & & 3.6 & 0.1 & 0.0 & & 66.5 & & 12.0 & & 370 & & 92.0 & & 0.8 \\
\hline p22 & 137 & 3.6 & 0.3 & 0.1 & 58.3 & 88.3 & 84.3 & 96.9 & 0.0 & 00 & 255.0 & 255.0 & 1.0 & 2.3 \\
\hline \multirow[t]{3}{*}{ p23 } & 485 & 4.9 & 0.2 & 0.0 & 56.6 & 64.2 & 84.9 & 96.1 & 0.0 & 00 & 255.0 & 255.0 & 2.0 & 1.0 \\
\hline & & \begin{tabular}{|l|}
4.9 \\
\end{tabular} & 0.2 & 0.0 & & 79.0 & & 101.8 & & 00 & & 255.0 & & 0.9 \\
\hline & 46 & 1.2 & \begin{tabular}{|l|}
0.2 \\
\end{tabular} & 0.0 & 55.6 & 66.6 & \begin{tabular}{|l|l|}
84.4 \\
\end{tabular} & 95.6 & 0.0 & 00 & 255.0 & 255.0 & 1.0 & 3.3 \\
\hline
\end{tabular}

P4, P6, P18, P19, P21, P23 patients have multi fractures in the bone that was shown in table 1

Table 2 Confusion Matrix

\begin{tabular}{|c|c|c|c|c|}
\hline \multicolumn{2}{|c|}{ TRUE CONDITION } & & & \\
\hline$\frac{\text { CONDITION }}{\text { POSITIVE }}$ & $\frac{\text { CONDITION }}{\text { NEGATIVE }}$ & $\begin{array}{c}\text { Prevalence } \\
0.73 \\
\end{array}$ & \multicolumn{2}{|c|}{ Accuracy } \\
\hline $\begin{array}{l}\text { True Positive } \\
41\end{array}$ & $\begin{array}{c}\text { False Positive } \\
3 \\
\end{array}$ & $\begin{array}{c}\text { Precision } \\
0.93 \\
\end{array}$ & \multicolumn{2}{|c|}{$\begin{array}{c}\text { False Discovery Rate } \\
0.06\end{array}$} \\
\hline $\begin{array}{c}\text { False Negative } \\
3\end{array}$ & $\begin{array}{l}\text { True Negative } \\
13\end{array}$ & $\begin{array}{c}\text { False Omission Rate } \\
0.18\end{array}$ & \multicolumn{2}{|c|}{$\begin{array}{c}\text { Negative Predicted } \\
\text { Value } \\
0.81 \\
\end{array}$} \\
\hline $\begin{array}{c}\text { True Positive Rate or } \\
\text { Sensitivity } \\
0.93\end{array}$ & $\begin{array}{c}\text { False Positive Rate } \\
0.18\end{array}$ & $\begin{array}{c}\text { Positive Likelihood } \\
\text { Ratio } \\
5.1\end{array}$ & \multirow{2}{*}{$\begin{array}{c}\text { Diagnostic } \\
\text { Odds } \\
\text { Ratio } \\
128\end{array}$} & \multirow[t]{2}{*}{$\begin{array}{c}\text { F1 Score } \\
0.93\end{array}$} \\
\hline $\begin{array}{c}\text { False Negative Rate } \\
0.06\end{array}$ & $\begin{array}{c}\text { True Negative Rate } \\
\text { or Specificity } \\
0.81\end{array}$ & $\begin{array}{c}\text { Negative Likelihood } \\
\text { Ratio } \\
0.07\end{array}$ & & \\
\hline
\end{tabular}

\section{CONCLUSION}

Identification of bone fracture from X-ray /CT images using advanced morphological technique is presented in this paper. It starts from image pre-processing and ends with the application of erode function. This technique is applied to 60 images and data that includes the area of fractured bone for 24 images is as shown above. The calculated statistical parameters are represented in a specific table 2 layout that determines the performance of the applied algorithm known as the confusion matrix. Analysis shows that the results obtained are satisfactory and accuracy $90 \%$ and sensitivity of $93 \%$ is achieved.

\section{REFERENCES}

1. Tandeep Kaur, Anupam Garg, "Bone Fracture Detection using Image Segmentation”, IJETT, June 2016, vol.36, pp 82-87

2. San Myint, Aung Soe Khaing, Hla Myo Tun, "Detecting Leg Bone Fracture in X-Ray Images", IJSTR, June 2016, vol.5,Issue 6, pp 140-144

3. Luis Nascimento, M.Graca Ruana, "Computer-Aided Bone Fracture Identification based on ultrasound images", IEEE, February 2015

4. Kamil Dimililer, "Intelligent Bone Fracture detection system”, ICSCCW, August 2017, vol.120, pp 260-267.

5. Malashree,G.Narayana Swamy, "Automatic Detection of Radius of Bone Fracture”, IJRET, June 2017, vol.4, Issue 6, pp 1796-1799.

6. Dhiraj B. Bhakare, Prajwal A.Jawalekar, Sumit D.Korde, "A Novel Approach for Bone Fracture Detection Using Image Processing”, IRJET, February 2018, vol. 5,Issue 2, pp 193-195.

7. Harmanpeet Kaur, Amit Jain, "Detection of Fractures in Orthopedic X-Ray Images", March 2017, vol. 8, pp 545-551

8. Cephas Paul Edward .V, Hilda Hepzibah S, "A Robust Approach for Detection of the type of Fracture from X-Ray Images", IJARCEE, March 2015, vol.4, Issue 3, pp 479-482

9. A.V. Manjari, G.S.N.S.Ajay Kamal, U.N.Subhadra Devi, Dr.M.Satyanarayana, "Detection and classification of Bone Fractures Using GLC Features", IJRASET, September 2017, vol.5, Issue 9, pp 581-586

10. Ozgur Ozturk, Hakan Kutucu, "Detection of bone fractures using image processing techniques and artificial networks", IEEE, September 2017

11. Irfan Karthik, "A study of Various Bone Fracture Detection Techniques", IJECS, May 2017, vol. 6, Issue 5, pp 21418-21423

12. Lu Ming, "Image Segmentation algorithm research and improvement", IEEE, August 2010, vol.5, pp 211-214.

13. Oishila Bandyopadhyay,Arindam Biswas, Bhargab B.Bhattacharya, "Long-Bone Fracture Detection in Digital X-ray Images Based on Concavity Index", IWCIA 2014, vol.8466, pp 212-223

14. A.Swarnalatha, Dr.M.Manikandan, "Review of Segmentation Techniques for Intravascular Ultrasound (IVUS) Images”, ICSCN, March 2017 